\title{
The Effect of the Underlying Malignancy on Short- and Medium- Term Survival of Critically III Patients Admitted to the Intensive Care Unit : A Retrospective Analysis Based on Propensity Score Matching
}

\author{
Zhennan Yuan \\ Cancer hospital/Chinese academy of medical sciences \\ Haijun Wang \\ Cancer hospital/Chinese academy of medical sciences \\ Yong Gao \\ Cancer hospital/Chinese academy of medical sciences \\ Shining Qu \\ Cancer hospital/Chinese academy of medical sciences \\ Chulin Huang \\ Cancer hospital/Chinese academy of medical sciences \\ Hao Wang \\ Cancer hospital/Chinese academy of medical sciences \\ Hao Zhang \\ Cancer hospital/Chinese academy of medical sciences \\ Quanhui Yang \\ Cancer hospital/Chinese academy of medical sciences \\ Xuezhong Xing ( $\nabla$ xueyujuanyanzi@163.com ) \\ Cancer hospital/Chinese academy of medical sciences
}

\section{Research article}

Keywords: Malignancy, Intensive care unit, Critical illness, Prognosis

Posted Date: September 17th, 2020

DOI: https://doi.org/10.21203/rs.3.rs-64412/v1

License: (c) (i) This work is licensed under a Creative Commons Attribution 4.0 International License. Read Full License

Version of Record: A version of this preprint was published at BMC Cancer on April 15th, 2021. See the published version at https://doi.org/10.1186/s12885-021-08152-5. 


\section{Abstract}

Background Advances in oncology led to a substantial increase in the number of patients requiring admission to the ICU. It is significant to confirm which cancer critical patients can benefit from the ICU care service like noncancer patients.

Methods An observational retrospective cohort study using intensive care unit (ICU) admissions of Medical Information Mart for Intensive Care III from the Beth Israel Deaconess Medical Center in Boston, MA, USA between 2001 and 2012 was conducted. Propensity score matching was used to reduce the imbalance between two matched cohorts. ICU patients with cancer were compared with those without cancer in terms of patients' characteristics and survival.

Results There were 38,508 adult patients admitted to ICUs during the period. The median age was 65 years (IQR, 52-77) and 8,308 $(21.8 \%)$ had an underlying malignancy diagnosis. The noncancer group had a significant survive advantage at the point of 28-day, 90day, 365-day and 1095-day after ICU admission compared with cancer group $(P<0.001$ for all) after PSM. Subgroup analysis showed that the diagnosis of malignancy didn't decrease 28-day and 90-day survive when patients' age $\geq 65$-year, patients in surgical intensive care unit or cardiac surgery recovery unit or traumatic surgical intensive care unit, elective admission, patients with renal replacement therapy or vasopressor support $(P>0.05$ for all).

Conclusions Malignancy is a common diagnosis among ICU patients. Patients without cancer have a survive advantage compared with patients with cancer in the short- and medium-term. However, in selected groups, cancer critical patients can benefit from the ICU care service like noncancer patients in the short-term.

\section{Background}

Historically, the presence of malignant disease had been a common reason for refusal of admission to intensive care unit (ICU) because of poor prognosis and high costs [1-3]. Survival of cancer patients has increased over the last three decades due to a greater awareness of early signs and better treatment $[4,5]$. Patients with cancer require ICU admission for postoperative care after major surgical resections, severe cancer- or chemo-radiation- or immunotherapy related complications, and concurrent severe acute illnesses[6, 7]. About 1 in 20 patients experienced a critical illness resulting in ICU admission within 2 years of cancer diagnosis and around one in seven patients admitted to general ICUs had a malignancy[6, 8, 9].

The outcome of cancer patients admitted to ICU is strongly dependent on the type of admission. Puxty et al.[10] studied 25,017 surgical admissions to general ICUs in the West of Scotland, and found that ICU and hospital mortality were lower in the group of ICU patients with cancer compared with noncancer patients. Bos et al.[11] reported on the characteristics and outcome of more than 15,000 cancer patients with an unplanned emergency admission to general ICUs in the National Intensive Care Evaluation registry between January 2007 and January 2011. They demonstrated that cancer patients have increased hospital mortality when compared with noncancer patients when admitted for medical reasons. When physicians decide to admit cancer patients to ICU, it should be kept in mind that the admitted cancer patients should be likely to benefit from ICU service. The challenge facing ICU physicians is to identify which cancer patients are likely to benefit from ICU care like noncancer patients. Most previously published studies didn't include a comparison group of patients without cancer; thus, it is difficult to determine the effect of cancer within the same ICU or hospital setting[11-13].

Our primary objective was to explore which critical ill patients with cancer can benefit from the ICU care like critical ill patients without cancer so that we can treat the diagnosis of malignancy as a chronic disease (such as hypertension, diabetes). We performed this study via available database of Medical Information Mart for Intensive Care III (MIMIC III) based on propensity score matching (PSM).

\section{Methods}

\section{Clinical database}

The MIMIC-III database is a large and freely available database comprising more than 40,000 patients in the ICU of the Beth Israel Deaconess Medical Center in Boston, MA, USA between 2001 and 2012. Our access to the database was approved after completion of the Collaborative Institutional Training Initiative (CITI program) web-based training course called "Data or Specimens Only research" (Record ID: 36067767).

\section{Data extraction}


Structured Query Language (SQL) with PostgreSQL (version 9.6) was used to extract data from MIMIC-III. Demographics (gender, age, ethnicity), ICU type, admission group (elective and emergency), reasons for ICU admission were extracted for adult patients ( $\geq$ 18 years) admitted to ICU. The severity of illness score was evaluated by the Sequential Organ Failure Assessment (SOFA) score[14], Simplified Acute Physiology Score II (SAPSII)[15], Logistic Organ Dysfunction Score (LODS)[16], Oxford Acute Severity of IIIness Score (OASIS)[17] and Acute Physiology Score III (APS III)[18]. For parameters of these five scoring systems, only data within $24 \mathrm{~h}$ after ICU admission were extracted and missing components for calculation were treated as normal (usually zero). Comorbidities were evaluated using the Elixhauser comorbidity system which scores a wide range of comorbid diseases and conditions according to the severity of organ decompensation and prognostic impact[19], the higher the score, the worse the patient's health. Other extracted data included mechanical ventilation (MV), vasopressor usage, renal replacement therapy (RRT), sepsis, hospital infection. Survival information was obtained from Social Security Death Index records. The endpoints of our study were 28-day, 90-day, 365-day and 1095-day survive after ICU admission.

\section{Population Selection Criteria}

Patients were not allowed to reenter the study after their first ICU admission. Enrolled cancer subjects were patients with diagnosis of malignancy according to International Classification of Diseases, Ninth Revision code (ICD-9). Suspected infection [20]was identified using the International Classification of Diseases, Ninth Revision, Clinical Modification codes (ICD-9-CM). The diagnosis of sepsis was based on the third international consensus definitions for sepsis and Septic Shock (Sepsis-3)[21]. The screening process of enrolled patients is shown in Fig. 1.

\section{Statistical analysis}

Categorical variables were described as the number and percentage, and their differences among groups were compared using Chisquared test. Noncontinuous variables and continuous variables that didn't follow normal distribution were expressed as median and interquartile range (IQR), and were analyzed with non-parametric methods (Mann-Whitney-Wilcoxon for two groups, Kruskal-Wallis for multi-groups). Continuous variables that followed normal distribution were expressed as mean and standard deviations, and t-test (two groups) or Analysis of Variance (ANOVA) (multiple groups) was used for these variables. Kaplan-Meier curves were used to calculate the survive rate and the log-rank test was used for comparisons between groups. The use of propensity score analysis aimed to reduce the imbalance between two matched cohorts. Standardized difference (SDD) before and after matching were plotted to show the effect of matching. In the cohort of propensity score-matched subjects, the SDD of all covariates between cancer group and noncancer group were $<10 \%$, which suggested that the propensity score matching appropriately adjusted for the initial selection bias. The bias in subgroups after successful PSM could also be considered as balanced[22]. A $P$ value $<0.05$ was considered statistically significant. Statistical analyses were performed using Stata, version 14.0 (Stata Corp).

\section{Results}

Characteristics of the patients with cancer and without cancer

During the study period, there were 38,508 patients whose age were older than 18 years admitted to ICUs in the Beth Israel Deaconess Medical Center in Boston, MA, USA between 2001 and 2012, of whom 16,715 (54.7\%) were male. The median age was 65 years (IQR, 52-77),and 8,308 (21.8\%) had an underlying malignancy diagnosis. Table 1 gives patient characteristics for admissions to ICU with and without a diagnosis of malignancy before and after PSM. ICU patients with cancer tended to be older than patients without cancer with median (IQR) age $70(60-79)$ VS $63(50-77)$ years $(P<0.001)$. The population without cancer admitted to ICU as an emergency was $85.9 \%$ (25,993 of 30,200 patients) in contrast to $78.1 \%$ (6,485 of 8,308 patients) of the population with cancer. Compared with patients with noncancer, patients with cancer were more likely to be admitted by surgical intensive care unit (SICU) (21.5\% VS 15.2\%) and medical intensive care unit (MICU) (42.0\% VS 33.6\%). The percentage of the noncancer patients in coronary care unit (CCU), cardiac surgery recovery unit (CSRU) and traumatic surgical intensive care unit (TSICU) were more than that of cancer patients. The admission reasons were otherwise similar between the cancer and noncancer groups with cardiovascular dysfunction, liver disorder, mental disorder, renal disorder, respiratory dysfunction, and coagulation dysfunction. Cancer patients tend to have high critical illness score compared with noncancer patients, SAPSII [39(26-46) VS 31(23-41), $P<0.001$ ], APSIII [40(30-53) VS 37(27-51), $P<0.001$ ]. The Elixhauser comorbidity index score of cancer patients was also significantly higher than noncancer patients $[9(2-17)$ VS $3(0-9), P<$ 0.001]. Respiratory support was the most common mode of support for both the cancer and noncancer groups at $40.3 \%$ (3,351 of 8,308 patients) and $47.2 \%$ ( 14,262 of 30,200 patients), respectively. Cardiovascular support was provided to $27.0 \%$ of the cancer group (2,240 of 8,308 patients) and $30.2 \%$ of the noncancer group $(9,114$ of 30,200 patients). Renal replacement therapy was not commonly provided in either group, but those patients in the cancer group had a lower prevalence of RRT [147 of 8,308 patients (1.8\%)] when compared with the noncancer group [889 of 30,200 patients $(2.9 \%), P<0.001]$. Patients with cancers were more likely to have a higher 
frequency of infection (42.7\% VS 38.6\%, $P<0.001)$ and sepsis (7.8\% VS 6.5\%, $P<0.001)$. After the propensity scores matched using $1: 1$, the covariates of the cancer group and noncancer group were balanced with a standard difference less than 5\% (Fig. 2). 
Table 1

Comparison of variables between Intensive care patients with cancer and without cancer before and after propensity score matched analysis

\begin{tabular}{|c|c|c|c|c|c|c|c|c|c|}
\hline \multirow[t]{2}{*}{ Variable } & \multirow{2}{*}{$\begin{array}{l}\text { ALL } \\
N=38,508\end{array}$} & \multicolumn{4}{|c|}{ Patients before PSM } & \multicolumn{4}{|c|}{ Patients after PSM } \\
\hline & & $\begin{array}{l}\text { Noncancer(N } \\
=30,200)\end{array}$ & $\begin{array}{l}\text { Cancer } \\
(\mathrm{N}=8,308)\end{array}$ & $\begin{array}{l}P \\
\text { Value }\end{array}$ & SDD & $\begin{array}{l}\text { Noncancer } \\
\mathrm{N}=7,474\end{array}$ & $\begin{array}{l}\text { Cancer } \\
\mathrm{N}=7,474\end{array}$ & $\begin{array}{l}P \\
\text { Value }\end{array}$ & SDD \\
\hline $\begin{array}{l}\text { Age, median } \\
(\mathrm{IQR}), \mathrm{Y}\end{array}$ & $65(52-77)$ & $63(50-77)$ & $70(60-79)$ & $\begin{array}{l}< \\
0.001\end{array}$ & 44.2 & $72(59-81)$ & $69(59-79)$ & 1.000 & -3.3 \\
\hline $\begin{array}{l}\text { Gender, No. } \\
(\%)\end{array}$ & & & & 0.545 & 0.8 & & & 0.921 & 0 \\
\hline Female & $16,715(43.4)$ & $13,133(43.5)$ & $3,582(43.1)$ & & & $3,276(43.8)$ & $3,282(43.9)$ & & \\
\hline Male & $21,783(56.6)$ & $17,067(56.5)$ & $4,726(56.8)$ & & & $4,198(56.2)$ & $4,192(56.1)$ & & \\
\hline $\begin{array}{l}\text { Ethnicity, No. } \\
(\%)\end{array}$ & & & & $\begin{array}{l}< \\
0.001\end{array}$ & 11.1 & & & 0.829 & 1.6 \\
\hline Black & $2,949(7.7)$ & $2,404(8.0)$ & $545(6.6)$ & & & $531(7.1)$ & $505(6.8)$ & & \\
\hline Asian & $911(2.4)$ & $667(2.2)$ & $244(2.9)$ & & & $195(2.6)$ & $207(2.8)$ & & \\
\hline White & $\begin{array}{l}27,468 \\
(71.3)\end{array}$ & $21,035(69.7)$ & $6,433(77.4)$ & & & $5,749(76.9)$ & $5,735(76.7)$ & & \\
\hline Hispanic & $1,254(3.3)$ & $1,095(3.6)$ & $159(1.9)$ & & & $154(2.1)$ & $154(2.1)$ & & \\
\hline Other & $5,926(15.4)$ & $4,999(16.6)$ & $927(11.2)$ & & & $845(11.3)$ & $873(11.7)$ & & \\
\hline $\begin{array}{l}\text { ICU type, No. } \\
(\%)\end{array}$ & & & & $\begin{array}{l}< \\
0.001\end{array}$ & 27.7 & & & 0.717 & 1.7 \\
\hline SICU & $6,366(16.5)$ & $4,578(15.2)$ & $1,788(21.5)$ & & & $1,528(20.4)$ & 1,499(20.1) & & \\
\hline MICU & $1,3634(35.4)$ & $10,148(33.6)$ & $3,486(42.0)$ & & & $3,090(41.3)$ & $3,064(41.0)$ & & \\
\hline $\mathrm{CCU}$ & $5,673(14.7)$ & $4,728(16.7)$ & $945(11.4)$ & & & $906(12.1)$ & $920(12.3)$ & & \\
\hline CSRU & $7,606(19.8)$ & $6,378(21.1)$ & $1,228(14.8)$ & & & $1,215(16.3)$ & $1,207(16.2)$ & & \\
\hline TSICU & $5,229(13.6)$ & $4,368(14.5)$ & $861(10.4)$ & & & $735(9.8)$ & 784(10.5) & & \\
\hline $\begin{array}{l}\text { Admission } \\
\text { group, No. (\%) }\end{array}$ & & & & $\begin{array}{l}< \\
0.001\end{array}$ & -20.2 & & & 0.764 & -0.4 \\
\hline Elective & $6,090(15.8)$ & $4,267(14.1)$ & $1,823(21.9)$ & & & $1,465(19.6)$ & $1,467(19.6)$ & & \\
\hline Emergency & $32,418(84.2)$ & $25,993(85.9)$ & $6,485(78.1)$ & & & $6,594(80.4)$ & $7,294(80.4)$ & & \\
\hline $\begin{array}{l}\text { Admission } \\
\text { reasons, No. } \\
(\%)\end{array}$ & & & & $\begin{array}{l}< \\
0.001\end{array}$ & -7.0 & & & 0.860 & 1.4 \\
\hline Cardiovascular & $17,528(45.5)$ & $13,726(45.5)$ & $3,802(45.8)$ & & & $3,419(45.8)$ & $3,416(45.7)$ & & \\
\hline Liver & $1,312(3.4)$ & $903(3.0)$ & 409(4.9) & & & $330(4.4)$ & $328(4.4)$ & & \\
\hline Mental & $4,118(10.7)$ & $3,300(10.9)$ & $818(9.9)$ & & & 783(10.5) & 771(10.3) & & \\
\hline Renal & $4,753(12.3)$ & $3,686(12.2)$ & $1,067(12.8)$ & & & $1,011(13.6)$ & $970(13.0)$ & & \\
\hline Respiratory & $6,905(18.0)$ & $5,478(18.2)$ & $1,427(17.2)$ & & & $1,239(16.6)$ & $1,288(17.2)$ & & \\
\hline
\end{tabular}

Abbreviations: Intensive care unit, ICU; the standardized differences, SDD; propensity score matching, PSM; IQR, 25-75\% interquartile range; Sequential Organ Failure Assessment, SOFA; Simplified Acute Physiology Score II, SAPSII; Logistic Organ Dysfunction Score, LODS; Oxford Acute Severity of Illness Score, OASIS; Acute Physiology Score, APSIII; Surgical intensive care unit, SICU; Medical intensive care unit, MICU; Coronary care unit, CCU; Cardiac surgery recovery unit, CSRU; Traumatic surgical intensive care unit, TSICU; mechanical ventilation, MV; renal replacement therapy, RRT. 


\begin{tabular}{|c|c|c|c|c|c|c|c|c|c|}
\hline \multirow{2}{*}{$\begin{array}{l}\text { Variable } \\
\text { Coagulation }\end{array}$} & \multirow{2}{*}{$\begin{array}{l}\text { ALL } \\
1,671(4.3)\end{array}$} & \multicolumn{4}{|c|}{ Patients before PSM } & \multicolumn{4}{|c|}{ Patients after PSM } \\
\hline & & $1,257(4.2)$ & $414(5.0)$ & & & $366(4.9)$ & $356(4.8)$ & & \\
\hline Other & $2,221(5.8)$ & $1,850(6.1)$ & $371(4.5)$ & & & $326(4.4)$ & $345(4.6)$ & & \\
\hline $\begin{array}{l}\text { SOFA, median } \\
\text { (IQR) }\end{array}$ & $3(2-5)$ & $3(2-5)$ & $3(2-5)$ & 0.001 & 2.5 & $3(2-6)$ & $3(2-6)$ & 0.622 & -0.9 \\
\hline $\begin{array}{l}\text { SAPS II, } \\
\text { median (IQR) }\end{array}$ & $24(33-92)$ & $31(23-41)$ & $39(26-46)$ & $\begin{array}{l}< \\
0.001\end{array}$ & 36.6 & $36(28-46)$ & $36(28-44)$ & 0.490 & -1.4 \\
\hline $\begin{array}{l}\text { APS III, median } \\
\text { (IQR) }\end{array}$ & $38(25-51)$ & $37(27-51)$ & $40(30-53)$ & $\begin{array}{l}< \\
0.001\end{array}$ & 11.4 & $40(29-54)$ & $39(30-52)$ & 0.462 & -2.0 \\
\hline $\begin{array}{l}\text { LODS, median } \\
\text { (IQR) }\end{array}$ & $3(2-5)$ & $3(2-5)$ & $3(2-5)$ & $\begin{array}{l}< \\
0.001\end{array}$ & 5.7 & $4(2-5)$ & $3(2-5)$ & 1.00 & -0.5 \\
\hline $\begin{array}{l}\text { OASIS, median } \\
\text { (IQR) }\end{array}$ & $30(25-37)$ & $30(25-37)$ & $30(25-37)$ & 0.293 & 1.2 & $31(25-37)$ & $31(25-37)$ & 0.09 & -1.4 \\
\hline $\begin{array}{l}\text { Elixhause } \\
\text { comorbidity, } \\
\text { median (IQR) }\end{array}$ & $4(0-12)$ & $3(0-9)$ & $9(2-17)$ & $<.001$ & 56.0 & $9(0-16)$ & $8(0-15)$ & 0.933 & -0.4 \\
\hline $\begin{array}{l}\text { RRT on first } \\
\text { day, No. (\%) }\end{array}$ & $1,036(2.7)$ & $889(2.9)$ & $147(1.8)$ & $\begin{array}{l}< \\
0.001\end{array}$ & -7.7 & $154(2.1)$ & $145(1.9)$ & 0.599 & -0.7 \\
\hline $\begin{array}{l}\text { MV on first } \\
\text { day, No. (\%) }\end{array}$ & $17,613(45.7)$ & $14,262(47.2)$ & $3,351(40.3)$ & $\begin{array}{l}< \\
0.001\end{array}$ & -13.9 & $3,051(40.8)$ & $3,084(41.3)$ & 0.583 & 1.1 \\
\hline $\begin{array}{l}\text { Vasopressor } \\
\text { administration } \\
\text { on first day, } \\
\text { No. (\%) }\end{array}$ & $11,354(29.5)$ & $9,114(30.2)$ & $2,240(27.0)$ & $\dot{0} 001$ & -7.1 & $2,094(28.0)$ & $2,076(27.8)$ & 0.743 & -1.1 \\
\hline Sepsis, No. (\%) & $2,601(6.8)$ & $1,951(6.5)$ & $650(7.8)$ & $\begin{array}{l}< \\
0.001\end{array}$ & 5.3 & $603(8.1)$ & 783(7.8) & 0.545 & -1.4 \\
\hline $\begin{array}{l}\text { Infection, No. } \\
(\%)\end{array}$ & $15,192(39.5)$ & $11,641(38.6)$ & $3,551(42.7)$ & $<.001$ & 8.5 & $3,164(42.3)$ & $2,196(42.8)$ & 0.597 & 1.1 \\
\hline \multicolumn{10}{|c|}{$\begin{array}{l}\text { Abbreviations: Intensive care unit, ICU; the standardized differences, SDD; propensity score matching, PSM; IQR, 25-75\% } \\
\text { interquartile range; Sequential Organ Failure Assessment, SOFA; Simplified Acute Physiology Score II, SAPSII; Logistic Organ } \\
\text { Dysfunction Score, LODS; Oxford Acute Severity of Illness Score, OASIS; Acute Physiology Score, APSIII; Surgical intensive care unit, } \\
\text { SICU; Medical intensive care unit, MICU; Coronary care unit, CCU; Cardiac surgery recovery unit, CSRU; Traumatic surgical intensive } \\
\text { care unit, TSICU; mechanical ventilation, MV; renal replacement therapy, RRT. }\end{array}$} \\
\hline
\end{tabular}

Outcomes of patients with cancer and without cancer before and after propensity score matched analysis (Table 2) 
Table 2

Outcomes of patients with cancer and without cancer before and after propensity score matched analysis

\begin{tabular}{|c|c|c|c|c|c|c|c|}
\hline \multirow[t]{2}{*}{ Outcome } & \multirow{2}{*}{$\begin{array}{l}\text { ALL } \\
n=38,508\end{array}$} & \multicolumn{3}{|c|}{ Patients before PSM } & \multicolumn{3}{|c|}{ Patients after PSM } \\
\hline & & $\begin{array}{l}\text { Noncancer } \\
\mathrm{N}=30,200\end{array}$ & $\begin{array}{l}\text { Cancer } \\
\mathrm{N}=8,308\end{array}$ & $\begin{array}{l}P \\
\text { Value }\end{array}$ & $\begin{array}{l}\text { Noncancer } \\
\mathrm{N}=7,474\end{array}$ & $\begin{array}{l}\text { Cancer } \\
\mathrm{N}=7,474\end{array}$ & $\begin{array}{l}P \\
\text { Value }\end{array}$ \\
\hline $\begin{array}{l}\text { LOS of ICU, median (IQR), } \\
\text { d }\end{array}$ & $2.1(1.2-4.1)$ & $2.1(1.2-4.1)$ & $2.1(1.2-3.9)$ & $\begin{array}{l}< \\
0.001\end{array}$ & $2.1(1.2-4.2)$ & $2.1(1.2-3.9)$ & $\begin{array}{l}< \\
0.001\end{array}$ \\
\hline $\begin{array}{l}\text { LOS of Hospital, median } \\
\text { (IQR), d }\end{array}$ & $6.9(4.0-11.9)$ & $6.8(4.0-11.8)$ & $\begin{array}{l}7.3(4.4- \\
12.2)\end{array}$ & $\begin{array}{l}< \\
0.001\end{array}$ & $\begin{array}{l}7.1(4.2- \\
12.6)\end{array}$ & $\begin{array}{l}7.2(4.3- \\
11.9)\end{array}$ & 0.774 \\
\hline 28-day survive, \% (95\%Cl) & $\begin{array}{l}86.6(86.2- \\
86.9)\end{array}$ & $\begin{array}{l}88.0(87.6- \\
88.4)\end{array}$ & $\begin{array}{l}81.3(80.5- \\
82.1)\end{array}$ & $\begin{array}{l}< \\
0.001\end{array}$ & $\begin{array}{l}84.9(84.0- \\
85.7)\end{array}$ & $\begin{array}{l}82.3(81.4- \\
83.1)\end{array}$ & $\begin{array}{l}< \\
0.001\end{array}$ \\
\hline 90-day survive, \% (95\%Cl) & $\begin{array}{l}81.8(81.4- \\
82.2)\end{array}$ & $\begin{array}{l}84.3(83.9- \\
84.7)\end{array}$ & $\begin{array}{l}72.9(72.0- \\
73.9)\end{array}$ & $\begin{array}{l}< \\
0.001\end{array}$ & $\begin{array}{l}79.2(78.3- \\
80.1)\end{array}$ & $\begin{array}{l}74.3(73.3- \\
75.3)\end{array}$ & $\begin{array}{l}< \\
0.001\end{array}$ \\
\hline $\begin{array}{l}\text { 365-day survive, \% } \\
(95 \% \mathrm{Cl})\end{array}$ & $\begin{array}{l}74.9(74.4- \\
75.3)\end{array}$ & $\begin{array}{l}79.1(78.7- \\
79.6)\end{array}$ & $\begin{array}{l}59.3(58.2- \\
60.3)\end{array}$ & $\begin{array}{l}< \\
0.001\end{array}$ & $\begin{array}{l}72.7(71.7- \\
73.7)\end{array}$ & $\begin{array}{l}61.4(60.3- \\
62.5)\end{array}$ & $\begin{array}{l}< \\
0.001\end{array}$ \\
\hline $\begin{array}{l}\text { 1,095-day survive, \% } \\
(95 \% \mathrm{Cl})\end{array}$ & $\begin{array}{l}67.3(66.9- \\
67.8)\end{array}$ & $\begin{array}{l}72.6(72.1- \\
73.1)\end{array}$ & $\begin{array}{l}48.3(47.3- \\
49.4)\end{array}$ & $<.001$ & $\begin{array}{l}64.6(63.5- \\
65.7)\end{array}$ & $\begin{array}{l}50.4(49.3- \\
51.6)\end{array}$ & $<.001$ \\
\hline
\end{tabular}

In the crude model, 28-day, 90-day, 365-day and 1095-day survive rate of noncancer group were $88.0 \%, 84.3 \%$, 79.1\% and 72.6\%, respectively, which were higher than the cancer group with $81.3 \%, 72.9 \%, 59.3 \%$ and $48.3 \%$, respectively. After PSM, the noncancer group still had a significant survive advantage at the point of 28-day, 90-day, 365-day and 1095-day after admission compared with cancer group ( $P<0.001$ for all) in the overall population (Fig. 3). Cancer patients had a similar length of ICU stay and slightly longer hospital stay (7.3-day VS 6.8-day, $P<0.001)$ compared with noncancer patients.

Subgroups analysis of short and medium-term survival of critically ill patients between with cancer and without cancer after PSM (Table 3) 
Table 3

28-day and 90-day Survive in Patients With and Without Cancer by Admission Features after propensity score matching

\begin{tabular}{|c|c|c|c|c|c|c|}
\hline \multirow[t]{2}{*}{ Variable } & \multicolumn{3}{|c|}{ 28-day survive, \% (95\% Cl) } & \multicolumn{3}{|c|}{ 90-day survive, \% (95\% Cl) } \\
\hline & Noncancer & Cancer & P Value & Noncancer & Cancer & $P$ Value \\
\hline \multicolumn{7}{|l|}{ Age } \\
\hline$<65$ & $92.2(91.1-93.1)$ & $84.3(82.9-85.6)$ & $<0.001$ & $90.2(88.9-91.2)$ & $76.7(75.1-78.3)$ & $<0.001$ \\
\hline$\geq 65$ & $80.9(79.7-82.0)$ & $81.1(79.9-82.2)$ & 0.697 & 73.3(72.0-74.5) & $72.9(71.6-74.1)$ & 0.791 \\
\hline \multicolumn{7}{|l|}{ Gender } \\
\hline Female & $83.6(82.2-84.8)$ & $80.2(78.8-81.5)$ & 0.001 & $78.2(76.8-79.6)$ & 72.3(70.8-73.8) & $<0.001$ \\
\hline Male & $85.9(84.8-86.9)$ & $83.9(82.8-85.0)$ & 0.012 & $80.0(78.8-81.2)$ & $75.9(74.5-77.1)$ & $<0.001$ \\
\hline \multicolumn{7}{|l|}{ Ethnicity } \\
\hline Black & $90.2(87.4-92.5)$ & $80.4(76.7-83.6)$ & $<0.001$ & $84.0(80.6-86.9)$ & $69.9(65.7-73.7)$ & $<0.001$ \\
\hline Asian & $85.2(79.3-89.4)$ & $80.6(74.6-85.4)$ & 0.215 & $80.0(73.7-85.0)$ & $71.0(64.3-76.7)$ & 0.036 \\
\hline White & $84.9(83.9-85.8)$ & $83.7(82.7-84.6)$ & 0.111 & $79.4(78.3-80.4)$ & $76.1(75.0-77.2)$ & $<0.001$ \\
\hline Hispanic & $90.3(84.4-94.0)$ & $86.4(79.9-90.9)$ & 0.293 & $87.7(81.3-92.0)$ & $74.7(67.0-80.8)$ & 0.005 \\
\hline Other & $80.2(77.4-82.8)$ & $73.9(70.8-76.7)$ & 0.002 & 73.5(70.4-76.3) & $66.0(62.7-69.0)$ & 0.001 \\
\hline \multicolumn{7}{|l|}{ ICU type } \\
\hline SICU & $84.4(82.5-86.2)$ & $86.1(84.3-87.8)$ & 0.161 & $78.6(76.5-80.6)$ & $78.6(76.4-80.6)$ & 0.843 \\
\hline MICU & $81.6(80.1-82.9)$ & 73.8(72.2-75.3) & $<0.001$ & $74.3(72.8-75.8)$ & $63.5(61.8-65.2)$ & $<0.001$ \\
\hline $\mathrm{CCU}$ & $81.9(79.2-84.3)$ & $85.7(83.2-87.8)$ & 0.022 & $77.4(74.5-80.0)$ & 78.5(75.7-81.0) & 0.409 \\
\hline CSRU & $95.5(94.1-96.5)$ & $94.4(92.9-95.5)$ & 0.218 & $93.2(91.6-94.5)$ & $91.2(89.5-92.7)$ & 0.076 \\
\hline TSICU & 85.7(83.0-88.1) & $85.6(82.9-87.9)$ & 0.978 & $80.3(77.2-83.0)$ & $77.3(74.2-80.1)$ & 0.216 \\
\hline \multicolumn{7}{|c|}{ Admission group } \\
\hline Elective & $96.8(95.7-97.5)$ & $96.5(95.5-97.4)$ & 0.774 & $94.1(92.7-95.2)$ & $94.2(92.9-95.3)$ & 0.864 \\
\hline Emergency & $81.8(80.8-82.7)$ & 78.7(77.6-79.7) & $<0.001$ & $75.6(74.5-76.7)$ & $69.5(68.3-70.6)$ & $<0.001$ \\
\hline \multicolumn{7}{|c|}{ Admission reasons } \\
\hline Cardiovascular & $86.2(85.0-87.3)$ & $83.4(82.1-84.6)$ & 0.003 & $82.0(80.7-83.2)$ & 76.5(75.0-77.9) & $<0.001$ \\
\hline Liver & $83.0(78.5-86.7)$ & 78.6(73.8-82.7) & 0.122 & $75.8(70.8-80.0)$ & $62.2(56.7-67.2)$ & $<0.001$ \\
\hline Mental & $81.1(78.2-83.7)$ & $77.7(74.6-80.5)$ & 0.090 & $74.0(70.7-76.9)$ & $68.7(65.3-71.8)$ & 0.020 \\
\hline Renal & $76.6(73.8-79.1)$ & 78.3(75.5-80.7) & 0.374 & $67.1(64.1-69.9)$ & $69.0(66.0-71.8)$ & 0.336 \\
\hline Respiratory & $87.4(85.4-89.1)$ & $84.4(82.3-86.3)$ & 0.003 & $82.2(80.0-84.3)$ & 78.7(76.4-80.9) & 0.026 \\
\hline Coagulation & $87.7083 .9-90.7$ & $78.9(74.2-82.9)$ & 0.001 & $79.5(75.0-83.0)$ & $68.3(63.2-72.8)$ & 0.001 \\
\hline Other & $94.8(91.8-96.7)$ & $91.6(88.1-94.1)$ & 0.116 & $92.3(88.9-94.8)$ & $81.7(77.2-85.4)$ & $<0.001$ \\
\hline \multicolumn{7}{|l|}{ SOFA, } \\
\hline$\varangle 3$ & $93.0(91.9-93.8)$ & $90.4(89.2-91.3)$ & 0.001 & $89.6(88.4-90.7)$ & $82.4(81.0-83.8)$ & 0.003 \\
\hline
\end{tabular}

Abbreviations: Sequential Organ Failure Assessment, SOFA; Simplified Acute Physiology Score II, SAPSII; Logistic Organ Dysfunction Score, LODS; Oxford Acute Severity of Illness Score, OASIS; Acute Physiology Score, APSIII; Surgical intensive care unit, SICU; Medical intensive care unit, MICU; Coronary care unit, CCU; Cardiac surgery recovery unit, CSRU; Traumatic surgical intensive care unit, TSICU; mechanical ventilation, MV; renal replacement therapy, RRT. 


\begin{tabular}{|c|c|c|c|c|c|c|}
\hline \multirow[t]{2}{*}{ Variable } & \multicolumn{3}{|c|}{ 28-day survive, \% (95\% Cl) } & \multicolumn{3}{|c|}{ 90-day survive, \% (95\% Cl) } \\
\hline & Noncancer & Cancer & P Value & Noncancer & Cancer & $P$ Value \\
\hline$\geq 3$ & $80.1(78.9-81.2)$ & $77.4(76.2-78.6)$ & 0.002 & $73.1(71.8-74.4)$ & $69.5(68.1-70.8)$ & $<0.001$ \\
\hline \multicolumn{7}{|l|}{ SAPS II } \\
\hline$\otimes 24$ & $98.1(97.1-98.7)$ & $96.7(95.4-97.7)$ & 0.044 & $97.3(96.2-98.1)$ & $93.2(91.5-94.6)$ & $<0.001$ \\
\hline$\geq 24$ & $82.4(81.5-83.4)$ & $80.1(79.1-81.1)$ & 0.001 & $75.9(74.8-76.9)$ & 71.5(70.4-72.6) & $<0.001$ \\
\hline \multicolumn{7}{|l|}{ APS III } \\
\hline$\bigotimes 38$ & $94.5(93.6-95.2)$ & $92.8(91.9-93.6)$ & 0.005 & $91.6(90.6-92.5)$ & $86.5(85.3-87.6)$ & $<0.001$ \\
\hline$\geq 38$ & $77.1(75.8-78.3)$ & $73.6(72.3-75.0)$ & 0.001 & $69.2(67.8-79.6)$ & $64.3(62.8-65.7)$ & $<0.001$ \\
\hline \multicolumn{7}{|l|}{ LODS } \\
\hline$凶 3$ & $93.9(92.8-94.7)$ & $90.9(89.8-92.0)$ & $<0.001$ & $90.8(89.6-91.8)$ & 83.5(82.0-84.8) & $<0.001$ \\
\hline$\geq 3$ & $80.2(79.0-81.3)$ & $77.8(76.6-78.9)$ & 0.006 & $73.2(72.0-74.5)$ & $69.6(68.3-70.8)$ & $<0.001$ \\
\hline \multicolumn{7}{|l|}{ OASIS } \\
\hline$\llbracket 30$ & $95.6(94.8-96.3)$ & $92.9(91.9-93.7)$ & $<0.001$ & $92.0(91.0-92.9)$ & $86.0(84.8-87.1)$ & $<0.001$ \\
\hline$\geq 30$ & 76.4(75.1-77.6) & $73.7(72.3-75.0)$ & 0.007 & $69.1(67.7-70.5)$ & $64.8(63.3-66.2)$ & $<0.001$ \\
\hline \multicolumn{7}{|c|}{ Elixhause comorbidity } \\
\hline$\varangle 4$ & $92.6(91.6-93.6)$ & $91.7(90.5-92.7)$ & 0.232 & $90.8(89.7-91.9)$ & $86.7(85.3-88.0)$ & $<0.001$ \\
\hline$\geq 4$ & $80.8(79.7-81.9)$ & $77.6(76.4-78.7)$ & $<0.001$ & 73.2(71.9-74.4) & $68.1(66.7-69.3)$ & $<0.001$ \\
\hline \multicolumn{7}{|l|}{ RRT } \\
\hline Yes & $69.5(61.3-76.3)$ & $71.0(62.9-77.7)$ & 0.951 & $61.7(53.5-68.8)$ & $63.5(55.1-70.7)$ & 0.930 \\
\hline No & $85.2(84.3-86.0)$ & $82.5(81.6-83.4)$ & $<0.001$ & $79.0(78.7-80.5)$ & $74.5(73.5-75.5)$ & $<0.001$ \\
\hline \multicolumn{7}{|l|}{$\mathrm{MV}$} \\
\hline Yes & $81.1(79.7-82.5)$ & 78.5(77.0-79.1) & 0.011 & $76.3(74.8-77.8)$ & $72.2(70.5-73.7)$ & $<0.001$ \\
\hline No & $87.4(86.1-88.4)$ & $84.8(83.9-86.0)$ & 0.001 & $81.2(80.0-82.3)$ & $75.8(74.5-77.1)$ & $<0.001$ \\
\hline \multicolumn{7}{|c|}{ Vasopressor } \\
\hline Yes & $79.1(77.3-80.8)$ & $77.5(75.7-79.2)$ & 0.254 & $74.0(72.0-75.8)$ & $72.3(70.3-74.2)$ & 0.249 \\
\hline No & $87.1(86.2-88.0)$ & $84,1(83.1-85.1)$ & $<0.001$ & $81.3(80.2-82.3)$ & $75.1(73.9-76.2)$ & $<0.001$ \\
\hline \multicolumn{7}{|l|}{ Sepsis } \\
\hline Yes & $64.0(60.0-67.7)$ & $51.3(47.2-55.3)$ & $<0.001$ & $52.4(48.3-56.3)$ & $41.7(37.7-45.7)$ & $<0.001$ \\
\hline No & $86.7(85.9-87.5)$ & $84.9(84.0-85.7)$ & 0.005 & $81.6(80.6-82.5)$ & $77.1(76.1-78.1)$ & $<0.001$ \\
\hline \multicolumn{7}{|l|}{ Infection } \\
\hline Yes & $78.9(77.5-80.3)$ & $75.2(73.6-76.6)$ & $<0.001$ & $70.0(68.3-71.5)$ & $64.8(63.1-66.4)$ & $<0.001$ \\
\hline No & $89.2(88.2-90.1)$ & 87.6(88.6) & 0.027 & $86.0(84.9-87.0)$ & $81.4(80.2-82.6)$ & $<0.001$ \\
\hline
\end{tabular}


The noncancer patients had survive advantage than cancer patients in MICU $(P<0.001$, Supp. Figure 1). However, when patients admitted by SICU, the survive advantage disappeared at the point of 28-day and 90-day ( $P>0.05$ for both, Supp. Figure 2$)$. Table 3 shows 28-day and 90-day survive in patients with and without cancer stratified according to distinct patient subgroups. Compared with noncancer group, 28-day and 90-day survive rate was lower in the cancer group when patient's age < 65, black, in MICU, emergency admission (Supp. Figure 3), admission because of cardiovascular or respiratory or coagulation dysfunction, Elixhauser comorbidity index score $\geq 4$, and patients without RRT or vasopressor support ( $P<0.05$ for all). 28-day and 90 -day survive rate showed no difference between cancer and noncancer group when patients' age $\geq 65$, patients in SICU or CSRU or TSICU, elective admission (Supp. Figure 4), patients with RRT or vasopressor support ( $P>0.05$ for all). Although when patients were Asian or White or Hispanic, admission because of liver or mental or renal disorder, Elixhauser comorbidity index score $<4$, patients with cancer showed no different 28-day survive compared with noncancer patients $(P>0.05$ for all), the survive advantage appeared at 90 -day survive for noncancer patients $(P<0.05$ for all). However, for patients in CCU, cancer group has higher 28-day survive rate than the noncancer group $(P=$ $0.022)$ and the survive advantage disappeared at the point of 90-day $(P=0.409)$.

\section{Discussion}

The interesting aspect of our study was the inclusion of consecutive admissions of cancer and non-cancer patients during the same time period. In the crude model, the higher survive rate was found in ICU patients with noncancer than those with cancer in the overall population at the point of 28-day, 90-day, 365-day and 1,095-day. Using a one-to-one propensity score matched analysis to address selection bias, we found that this difference was narrowed though noncancer patients still had a slight survival advantage. In this study, we accepted five criticality scores to evaluate the illness of patients. The criticality scores of cancer patients were higher than those of noncancer patients. No matter in the group with high critical illness score or the group with low critical illness score, the 28-day and 90-day survive of cancer patients was significantly worse than that of noncancer patients $(P<0.05$ for both). ICU patients with cancer tended to be older than patients without cancer and cancer patients usually had a worse outcome when patients' age $>65$. However, the poor outcome wasn't associated with the diagnosis of malignancy.

To further subgroup analysis of 28-day and 90-day survive associated with cancer diagnosis, we found that the diagnosis of the malignancy didn't decrease the 28-day and 90-day survival rates in selected groups. When patients admitted by MICU, 28-day and 90day survive was higher in noncancer patients than cancer patients. However, cancer patients who were admitted by SICU had similar outcomes compared with those of noncancer patients. This finding is in keeping with those of previous studies[10, 11]. For cancer patients admitted to MICU, the stage was usually late [13]and the cancer related therapy may lead to immunosuppressive status[23], so the early prognosis was usually poor. However, for patients in SICU, the stage was usually early[13] and the tumor resection have reduced most of the tumor load, so the diagnosis of malignancy had few effects on their short-term prognosis. So we can concluded that short-term survive rates especially differed between cancer and non-cancer patients admitted to ICU because of medical reasons, whereas differences between cancer and non-cancer patients were either not existing or modest in SICU.

Emergency or elective admissions often affect the prognosis of patients in the ICU. In the present study, when patients admitted as elective admission, it showed no difference of survive at the point of 28-day and 90-day between cancer and noncancer patients although the difference appeared at the point of 365-day. When patients admitted as emergency, the noncancer patients has a survive advantage within 1095 days after ICU admission. Bos et al.[11] also reported the similar outcome. They demonstrated that emergency ICU admission is associated with a survive advantage in patients with noncancer. It is associated with a higher incidence of acute comorbidity and a greater severity of illness on admission in the cancer group[11].

In the present study, patients with cancer had a greater need for intensive support (eg, MV, vasopressor administration, and RRT), which was consistent with previous literature[24]. In patients with MV support, we found that noncancer patients had survive advantage at the point of 28-day and 90-day after ICU admission. Hsiue et al.[25]compared 518 patients with solid cancers with 1362 non-cancer patients receiving MV admitted to ICU between 2012 and 2014. They found that the 28-day and 90-day mortality rates were higher in cancer patients than in noncancer patients ( $45.2 \%$ vs. $29.4 \%$, and $65.6 \%$ vs. $37.7 \%$, respectively, both $P<0.001)$. It remains controversial to start RRT for acute renal failure in critically ill patients with cancer because of the poor outcome and high costs[26-31]. However, this controversy lacked large sample research in previous literature. In our study, for patients in need of RRT, the diagnosis of malignancy didn't decrease their 28-day and 90-day survive in this study. Benoit et al. [3] and Maccariello et al. [32] also demonstrated that though the early prognosis of cancer patients with acute renal failure in need of RRT is poor, the malignancy itself is not an independent risk factor that affects the prognosis. Cancer patients with shock admitted to ICU usually have a poor prognosis[33]. Previous study reported the in-hospital mortality rate of cancer patients with sepsis shock seemed to be higher than that of noncancer

Page $10 / 17$ 
patients[34-36]. However, they did not include a control group of septic shock patients without cancer. In the present study, when cancer patients with RRT or vasopressor support admitted to ICU, they a had worse short-term outcome, but the poor outcome was not caused by the diagnosis of malignancy.

It has found chronic disease burden to be significantly associated with short-term outcome[37, 38]. We accepted a single numeric score based on the Elixhauser comorbidity index to describe the chronic health status of patients[19]. Cancer patients seems to be with more chronic comorbidities than noncancer patients. In this study, for those patients with poor chronic health or limited functional status, the diagnosis of malignancy had been demonstrated to be associated with poorer 28-day survival. While for patients with a better health status (Elixhauser comorbidity score < 4), the survive difference disappeared at the point of 28-day though the noncancer group still had survive advantage at the point of 90 -day. So the diagnosis of malignancy have a more effect on patients with much comorbidities.

Patients with cancer diagnosis were more likely to have infections or sepsis compared with those noncancer patients in this study. Cancer has been reported in about $17 \%$ associated with sepsis in MICU[39]. Sepsis is one of the major causes of ICU admission for cancer patients[7] and is an important cause of hospital mortality and morbidity[9]. Treatment of cancer has contributed to a growing number of immunocompromised patients with an increased incidence of hospital infections; immunosuppression can result in a greater use of antibiotics and more infections associated with multi-resistant microorganisms[23]. In our study, cancer patients with hospital infection or sepsis had worse prognosis than noncancer patients with hospital infection or sepsis. Therefore, for patients with malignancy, we should pay attention to the status of potential infections.

The strength of the present study includes the low confounding bias due to equivalence of covariates between the two study groups in the propensity score-matched cohort. The propensity score method creates a model that reflects the effects of risk factors on the exposure. Matching using a single summary variable that predicts the probability of being exposed as a function of the confounders creates a statistical model with fewer assumptions and may lead to improved estimates of exposure effects in some settings[22]. This study also included a comparison group of patients without cancer to determine effect of cancer within the same ICU setting. Our study also had shortcomings, firstly, the cancer stage is not available, which may be a factor that affects the early prognosis of the patients. Secondly, the time of diagnosis of malignancy was not consistent, which may be more than 2 years earlier before their ICU admission. Those patients with more than 5-years tumor free survive can be considered as cured completely.

\section{Conclusions}

Advances in oncology led to a substantial increase in the number of patients requiring admission to the ICU. In the overall population, noncancer patients had a survive advantage of short- and medium-term. When patients' age $\geq 65$ years, patients in SICU or CSRU or TSICU, elective admission, patients with RRT or vasopressor support, the diagnosis of malignancy didn't decrease their short-term survive and cancer patients can benefit from ICU intensive care like noncancer patients. These findings can help us make cancerrelated prognosis judgments and make corresponding clinical decisions.

\section{Abbreviations}

Intensive care unit, ICU;

the standardized differences, SDD;

propensity score matching, PSM;

IQR, 25-75\% interquartile range;

Sequential Organ Failure Assessment, SOFA;

Simplified Acute Physiology Score II, SAPSIl;

Logistic Organ Dysfunction Score, LODS;

Oxford Acute Severity of Illness Score, OASIS;

Acute Physiology Score, APSIII;

Page $11 / 17$ 
Surgical intensive care unit, SICU;

Medical intensive care unit, MICU;

Coronary care unit, CCU;

Cardiac surgery recovery unit, CSRU;

Traumatic surgical intensive care unit, TSICU;

mechanical ventilation, MV;

renal replacement therapy, RRT.

\section{Declarations}

\section{Ethics approval and consent to participate}

This study used a public de-identified database thus informed consent and approval of the Institutional Review Board was waived.

\section{Competing interests}

The authors declare that they have no competing interests.

\section{Availability of data and materials}

The datasets used and/or analyzed during the current study are available from the corresponding author on reasonable request.

\section{Funding}

None

\section{Authors' contributions}

(I) Conception and design: Xuezhong-Xing.

(II) Administrative support: Xuezhong-Xing.

(III) Provision of study materials or patients: Zhennan-Yuan, Yong Gao, Shining-Qu, Chulin-Huang, Quanhui-Yang.

(IV) Collection and assembly of data: Zhennan-Yuan and Haijun-Wang.

(V) Data analysis and interpretation: Zhennan-Yuan, Hao Zhang and Hao Wang.

(VI) Manuscript writing: All authors.

(VII) Final approval of manuscript: All authors.

\section{Acknowledgement}

None

\section{References}

1. Darmon M, Azoulay E. Critical care management of cancer patients: cause for optimism and need for objectivity. Curr Opin Oncol. 2009;21:318-26.

2. Price KJ, Thall PF, Kish SK, Shannon VR, Andersson BS. Prognostic indicators for blood and marrow transplant patients admitted to an intensive care unit. Am J Respir Crit Care Med. 1998;158:876-84. 
3. Benoit DD, Hoste EA, Depuydt PO, Offner FC, Lameire NH, Vandewoude KH, Dhondt AW, Noens LA, Decruyenaere JM. Outcome in critically ill medical patients treated with renal replacement therapy for acute renal failure: comparison between patients with and those without haematological malignancies. Nephrology, dialysis, transplantation: official publication of the European Dialysis and Transplant Association -. European Renal Association. 2005;20:552-8.

4. Siegel RL, Miller KD, Jemal A. Cancer statistics, 2018. Cancer J Clin. 2018;68:7-30.

5. Cai Z, Liu Q. (2019) Understanding the Global Cancer Statistics 2018: implications for cancer control. Sci China Life Sci.

6. Puxty K, McLoone P, Quasim T, Sloan B, Kinsella J, Morrison DS. Risk of Critical Illness Among Patients With Solid Cancers: A Population-Based Observational Study. JAMA Oncol. 2015;1:1078-85.

7. Hawari Fl, Nazer LH, Addassi A, Rimawi D, Jamal K. Predictors of ICU Admission in Patients With Cancer and the Related Characteristics and Outcomes: A 5-Year Registry-Based Study. Critical care medicine. 2016;44:548-53.

8. Bos MM, Verburg IW, Dumaij I, Stouthard J, Nortier JW, Richel D, van der Zwan EP, de Keizer NF, de Jonge E. Intensive care admission of cancer patients: a comparative analysis. Cancer Med. 2015;4:966-76.

9. Taccone FS, Artigas AA, Sprung CL, Moreno R, Sakr Y, Vincent JL. Characteristics and outcomes of cancer patients in European ICUs. Crit Care. 2009;13:R15.

10. Puxty K, McLoone P, Quasim T, Sloan B, Kinsella J, Morrison DS. Characteristics and Outcomes of Surgical Patients With Solid Cancers Admitted to the Intensive Care Unit. JAMA Surgery. 2018;153:834.

11. Bos MM, de Keizer NF, Meynaar IA, Bakhshi-Raiez F, de Jonge E. Outcomes of cancer patients after unplanned admission to general intensive care units. Acta Oncol. 2012;51:897-905.

12. Bos MM, Bakhshi-Raiez F, Dekker JW, de Keizer NF, de Jonge E. Outcomes of intensive care unit admissions after elective cancer surgery. European journal of surgical oncology: the journal of the European Society of Surgical Oncology the British Association of Surgical Oncology. 2013;39:584-92.

13. Soares M, Caruso P, Silva E, Teles JM, Lobo SM, Friedman G, Dal Pizzol F, Mello PV, Bozza FA, Silva UV, Torelly AP, Knibel MF, Rezende E, Netto JJ, Piras C, Castro A, Ferreira BS, Rea-Neto A, Olmedo PB, Salluh JI. Brazilian Research in Intensive Care N, (2010) Characteristics and outcomes of patients with cancer requiring admission to intensive care units: a prospective multicenter study. Critical care medicine 38: 9-15.

14. Vincent JL, de Mendonca A, Cantraine F, Moreno R, Takala J, Suter PM, Sprung CL, Colardyn F, Blecher S. Use of the SOFA score to assess the incidence of organ dysfunction/failure in intensive care units: results of a multicenter, prospective study. Working group on "sepsis-related problems" of the European Society of Intensive Care Medicine. Critical care medicine. 1998;26:1793-800.

15. Le Gall JR, Lemeshow S, Saulnier F. A new Simplified Acute Physiology Score (SAPS II) based on a European/North American multicenter study. Jama. 1993;270:2957-63.

16. Le Gall JR, Klar J, Lemeshow S, Saulnier F, Alberti C, Artigas A, Teres D. The Logistic Organ Dysfunction system. A new way to assess organ dysfunction in the intensive care unit. ICU Scoring Group Jama. 1996;276:802-10.

17. Johnson AE, Kramer AA, Clifford GD. A new severity of illness scale using a subset of Acute Physiology And Chronic Health Evaluation data elements shows comparable predictive accuracy. Critical care medicine. 2013;41:1711-8.

18. Pollack MM, Patel KM, Ruttimann UE. The Pediatric Risk of Mortality III-Acute Physiology Score (PRISM III-APS): a method of assessing physiologic instability for pediatric intensive care unit patients. J Pediatr. 1997;131:575-81.

19. van Walraven C, Austin PC, Jennings A, Quan H, Forster AJ. A modification of the Elixhauser comorbidity measures into a point system for hospital death using administrative data. Med Care. 2009;47:626-33.

20. Angus DC, Linde-Zwirble WT, Lidicker J, Clermont G, Carcillo J, Pinsky MR. Epidemiology of severe sepsis in the United States: analysis of incidence, outcome, and associated costs of care. Critical care medicine. 2001;29:1303-10.

21. Seymour CW, Liu VX, Iwashyna TJ, Brunkhorst FM, Rea TD, Scherag A, Rubenfeld G, Kahn JM, Shankar-Hari M, Singer M, Deutschman CS, Escobar GJ, Angus DC. Assessment of Clinical Criteria for Sepsis: For the Third International Consensus Definitions for Sepsis and Septic Shock (Sepsis-3). Jama. 2016;315:762-74.

22. Wang SV, Jin Y, Fireman B, Gruber S, He M, Wyss R, Shin H, Ma Y, Keeton S, Karami S, Major JM, Schneeweiss S, Gagne JJ. Relative Performance of Propensity Score Matching Strategies for Subgroup Analyses. Am J Epidemiol. 2018;187:1799-807.

23. Berghmans T, Crokaert F, Markiewicz E, Sculier JP. Epidemiology of infections in the adult medical intensive care unit of a cancer hospital. Supportive care in cancer: official journal of the Multinational Association of Supportive Care in Cancer. 1997;5:234-40. 
24. Rosa RG, Tonietto TF, Duso BA, Maccari JG, de Oliveira RP, Rutzen W, Madeira L, Ascoli A, Hessler R, Morandi P, Cremonese RV, Neto FLD, Tagliari L, de Campos Balzano P, Barth JHD, Teixeira C. Mortality of Adult Critically III Subjects With Cancer. Respir Care. 2017;62:615-22.

25. Hsiue EH-C, Lee P-L, Chen Y-H, Wu T-H, Cheng C-F, Cheng K-M, Yang P-C, Chen H-W, Lin P-Y, Chiang D-L, Wu H-D, Yang JC-H, Yu C-J. Weaning outcome of solid cancer patients requiring mechanical ventilation in the intensive care unit. J Formos Med Assoc. 2019;118:995-1004.

26. Brivet FG, Kleinknecht DJ, Loirat P, Landais PJ. (1996) Acute renal failure in intensive care units-causes, outcome, and prognostic factors of hospital mortality; a prospective, multicenter study. French Study Group on Acute Renal Failure. Critical care medicine 24: $192-198$.

27. Masoomi H, Carmichael JC, Dolich M, Mills S, Ketana N, Pigazzi A, Stamos MJ. Predictive factors of acute renal failure in colon and rectal surgery. Am Surg. 2012;78:1019-23.

28. Gumbert SD, Kork F, Jackson ML, Vanga N, Ghebremichael SJ, Wang CY, Eltzschig HK. Perioperative Acute Kidney Injury. Anesthesiology. 2020;132:180-204.

29. Audisio RA. Risk factors for morbidity and mortality after colectomy for colon cancer. Tech Coloproctol. 2001;5:177-9.

30. Uchino S, Kellum JA, Bellomo R, Doig GS, Morimatsu H, Morgera S, Schetz M, Tan I, Bouman C, Macedo E, Gibney N, Tolwani A, Ronco C. Beginning, Ending Supportive Therapy for the Kidney I, (2005) Acute renal failure in critically ill patients: a multinational, multicenter study. Jama 294: 813-818.

31. Negi S, Koreeda D, Kobayashi S, Yano T, Tatsuta K, Mima T, Shigematsu T, Ohya M. Acute kidney injury: Epidemiology, outcomes, complications, and therapeutic strategies. Semin Dial. 2018;31:519-27.

32. Maccariello E, Valente C, Nogueira L, Bonomo H Jr, Ismael M, Machado JE, Baldotto F, Godinho M, Rocha E, Soares M. Outcomes of cancer and non-cancer patients with acute kidney injury and need of renal replacement therapy admitted to general intensive care units. Nephrology, dialysis, transplantation: official publication of the European Dialysis and Transplant Association European Renal Association. 2011;26:537-43.

33. Biskup E, Cai F, Vetter M, Marsch S. Oncological patients in the intensive care unit: prognosis, decision-making, therapies and endof-life care. Swiss Med Wkly. 2017;147:w14481.

34. Labelle A, Juang P, Reichley R, Micek S, Hoffmann J, Hoban A, Hampton N, Kollef M. The determinants of hospital mortality among patients with septic shock receiving appropriate initial antibiotic treatment*. Critical care medicine. 2012;40:2016-21.

35. Kaukonen KM, Bailey M, Suzuki S, Pilcher D, Bellomo R. Mortality related to severe sepsis and septic shock among critically ill patients in Australia and New Zealand, 2000-2012. Jama. 2014;311:1308-16.

36. Lee MR, Lai CL, Chan KA. Intensive Care Unit Admission and Survival in Stage IV Cancer Patients with Septic Shock: A PopulationBased Cohort Study. J Cancer. 2019;10:3179-87.

37. Poses RM, McClish DK, Smith WR, Bekes C, Scott WE. Prediction of survival of critically ill patients by admission comorbidity. J Clin Epidemiol. 1996;49:743-7.

38. Puxty K, McLoone P, Quasim T, Kinsella J, Morrison D. Survival in solid cancer patients following intensive care unit admission. Intensive care medicine. 2014;40:1409-28.

39. Danai PA, Moss M, Mannino DM, Martin GS. The epidemiology of sepsis in patients with malignancy. Chest. 2006;129:1432-40.

\section{Figures}




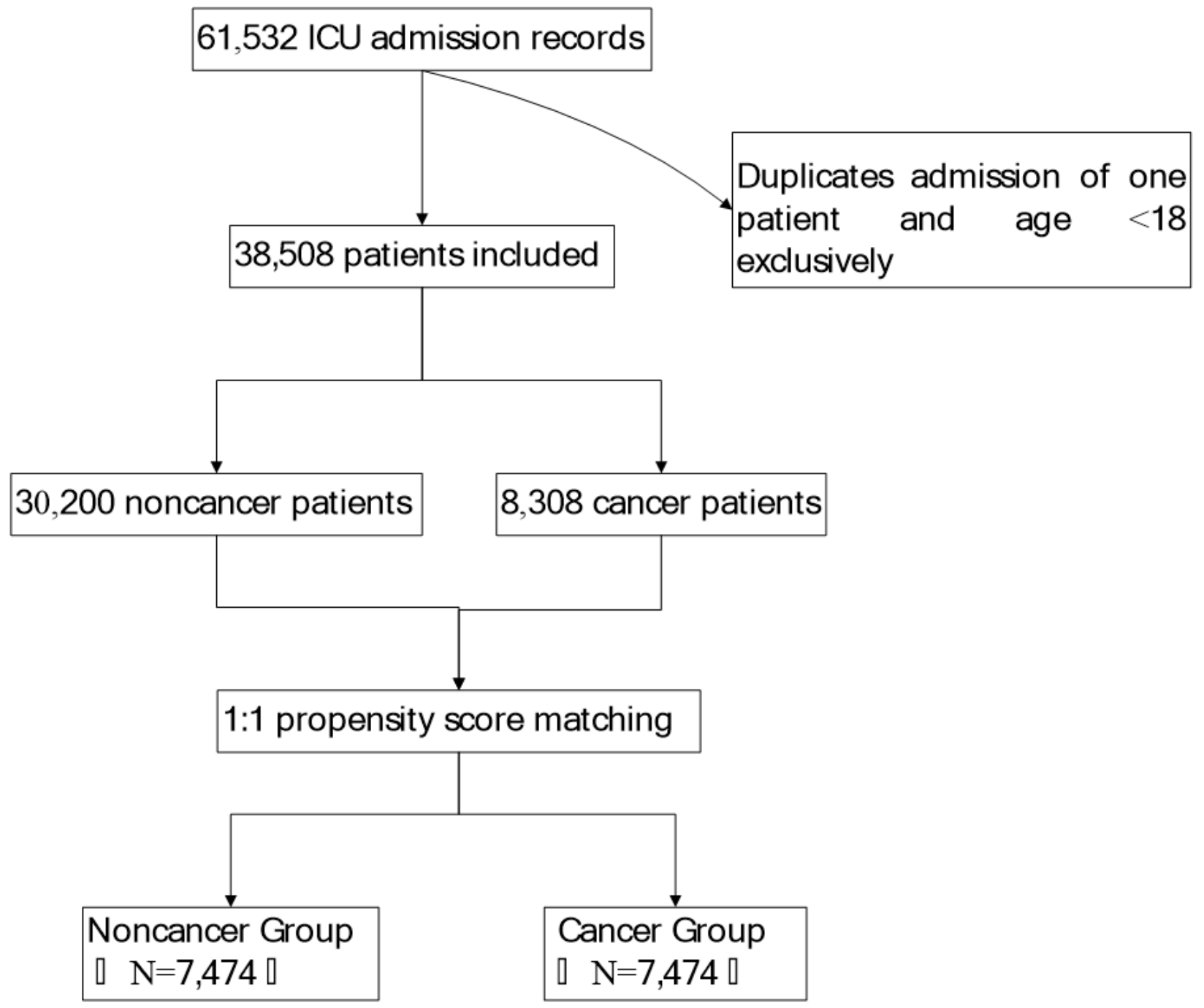

Figure 1

Flow plot of data extraction and filtration from the MIMIC-III database 


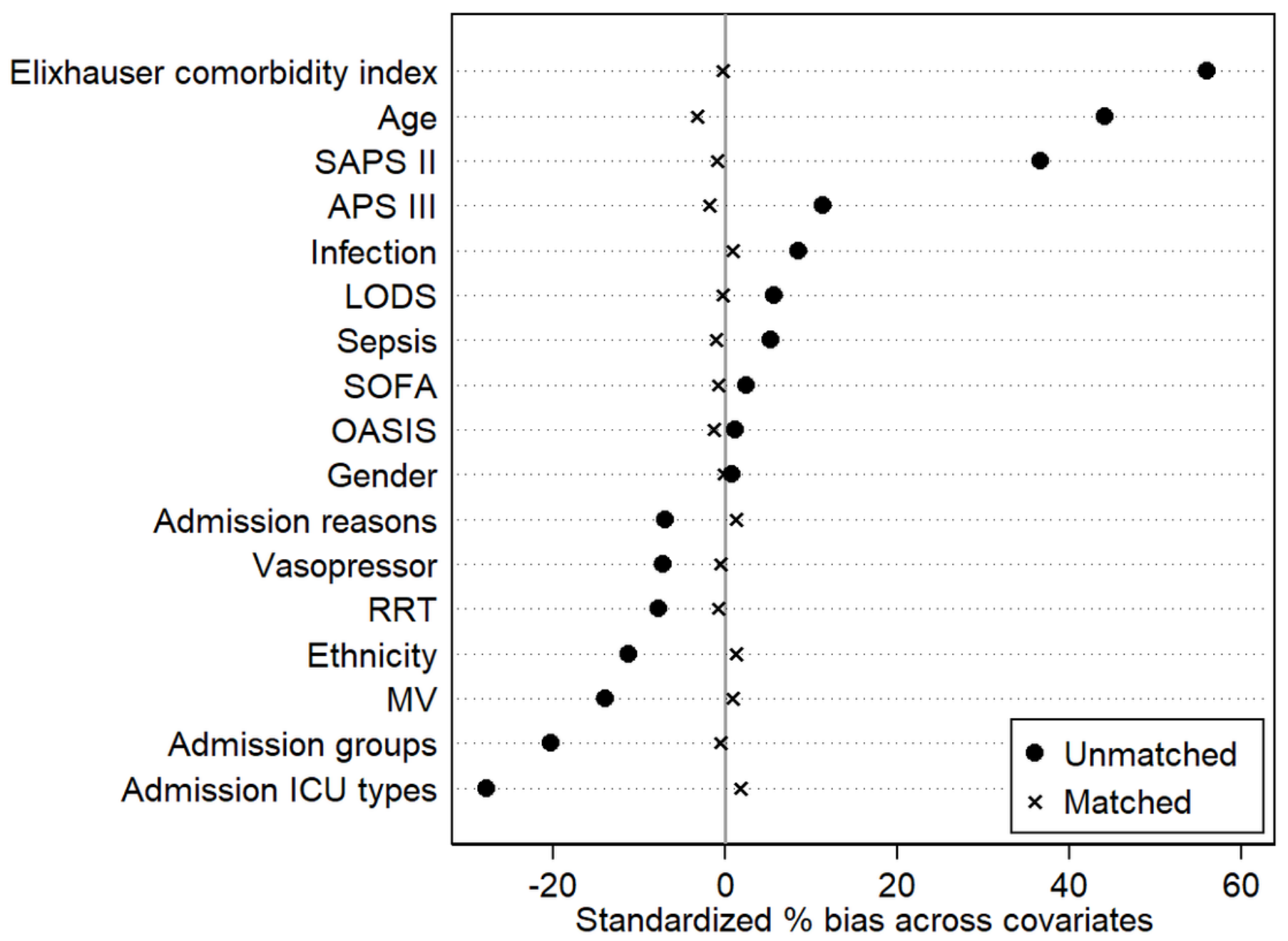

Figure 2

Balance of covariates between critical care patients with and without cancer and before and after propensity score matching. (Abbreviations: Sequential Organ Failure Assessment, SOFA; Simplified Acute Physiology Score II, SAPSIl; Logistic Organ Dysfunction Score, LODS; Oxford Acute Severity of IIIness Score, OASIS; Acute Physiology Score, APSIII; intensive care unit, ICU; mechanical ventilation, MV; renal replacement therapy, RRT) 


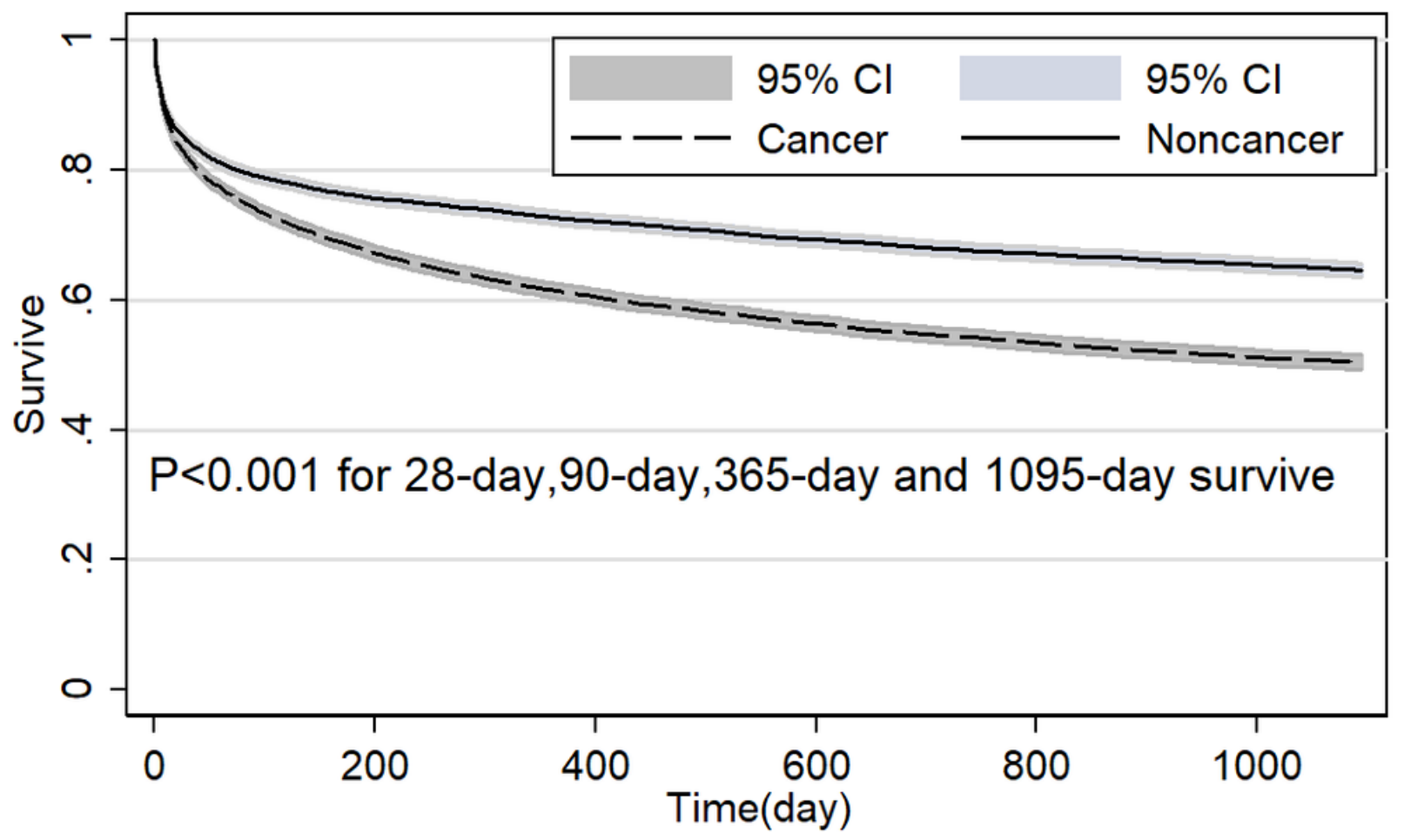

Number at risk

Cancer 7474 Noncancer 7474

5024

4525

4213

3994

3825 5656

5390

5180

5020

4893

Figure 3

Comparison of Kaplan-Meier survival curves between critical care subjects with and without cancer in overall population after propensity score matching

\section{Supplementary Files}

This is a list of supplementary files associated with this preprint. Click to download.

- Supp.docx 